Proc. 8th IEEE Intl. Symp. on Personal, Indoor, and Mobile Radio Comm., (PIMRC), pp. 62-67, Helsinki, Finland, September 1997.

\title{
Computationally Efficient Multiuser Detectors
}

\author{
Suman Das Joseph R. Cavallaro Behnaam Aazhang \\ Department of Electrical and Computer Engineering,Rice University \\ MS 366, Houston, Texas 77005-1892 \\ Phone (713)527-8750x2899 Fax:(713)-524-5237 \\ Email: \{suman,cavallar,aaz\}@rice.edu
}

\begin{abstract}
CDMA is becoming an increasingly popular multiplexing scheme in wireless communication and this has necessitated the development of efficient detection techniques. The exponential complexity of the optimal detector on one end and inferior performance of conventional single-user detector at the other have led to the development of suboptimal multiuser detectors with lower complexity. Most of these detection techniques involve solution of a linear system. In their naive implementation this requires $O\left(n^{3}\right)$ operations in the size of the matrix. This cost can be reduced if we move towards modern iterative techniques for solution of the system. However maximum benefit can be achieved if we fully exploit the structure of the system. In this paper wo hove proposed several methods of reducing the computational complexity utilizing the above ideas. We have also come up with algorithms which computationally can achieve the lower bound in complexity.
\end{abstract}

\section{INTRODUCTION}

In multiple access communication systems a large number of users share a common communication channel. Due to its superior capacity and channel utilization, Code-division multiple-access (CDMA) is rapidly gaining popularity over other multiplexing schemes in wireless communication. While in traditional multiple-access schemes, like FDMA and TDMA, users are distinguished by allocation of orthogonal frequency und time slots, in CDMA, users are distinguished in the code-space.

In direct-sequence CDMA (DS-CDMA) each user's information bits are modulated by the distinct individual code and the receiver receives a signal which is the composite sum of signals of all the users. The detector, which has the perfect knowledge of each user's individual code and the delays (in the asynchronous case), tries to extract the information bits from the received signal.

In a single-user environment, under the additive-white Gaussian noise (AWGN) assumption, the optimal detector detects the information bits by passing the received signal through a filter, matched to the user code. The conventional DS-CDMA detector extends the idea of single user detection to the multi-user case by passing the received signal through a bank of code-matched filters.

In his seminal paper [15] Verdu showed that this single-user detection scheme is sub-optimal. In the multi-user system the optimal detector is the Maximum r.ikeliband Eatimate (MLE.) detector. This detector far outperforms the conventional matched filter detector. However this detector has exponential complexity.

As a compromise, attempts have been made since then to come up with various suboptimal detectors which perform better than the conventional detector but have less computational complexity than the MLE detector. Most of these detectors solve a linear system of equations. Naive implementation of these algorithms will require a $O\left(n^{3}\right)$ computational complexity. However these methods of solution do not exploit the structure in the system. Modern numerical methods have provided us with a lot of insights to exploit the structure of the system which may greatly reduce the computational effort. In this paper we have developed varions algorithms which exploit the structure to reduce the complexity. We have determined the lower bound for computational complexity of these algorithms and propose two algorithms which can achieve this lower bound.

The paper is organized as follows.In the following section we will describe the system. In section 3 we discuss the various suboptimal detectors and introduce iterative techniques to solve the system. Section 4 deals with special structures to be used for iterative techniques. Section 5 lists two algorithms which are optimal in complexity. Section 6 draws the conclusion and points to future directions.

\section{SYSTEM DESCRIPTION}

We will consider an asynchronous system with $K$ users. We will also assume that each user sends information of block length $N$. Let the $i^{\text {th }}$ user have a signature waveform given by $s_{i}$, which extends over the symbol period $[0, T]$. Without loss of generality we will assume that the users are ordered in ascending order of their delays

$$
0=\tau_{1} \leq \tau_{2} \leq \cdots \leq \tau_{K}
$$

We will also assume that each of the user delays are known to the detector. If the user amplitudes are given by

$$
A=\left[A_{1}, A_{2}, \cdots, A_{K}\right]
$$

and the data stream for the $k^{t h}$ user is given by

$$
b_{k}^{T}=\left[b_{k}(1), b_{k}(2), \cdots, b_{k}(N)\right]
$$


then the cumulative signal received by the detector is given as

$$
r(t)=\sum_{k=1}^{K} \sum_{i=1}^{N} A_{k} b_{k}(i) s_{k}\left(t-i T-n_{k}\right)+n(t),
$$

where $n(t)$ is AWGN.

If this received signal is passed through a bank of code matched filters then the output of the filter bank can be expressed [11] in the vector form as

$$
y=R_{N} b+\eta
$$

where $R_{N}$ is the code-correlation matrix of the form

$$
R_{N}=\left[\begin{array}{cccccc}
R(0) & R^{t}(1) & 0 & \cdots & 0 & 0 \\
R(1) & R(0) & R^{t}(1) & 0 & \cdots & 0 \\
\vdots & \vdots & \vdots & \vdots & \vdots & \vdots \\
0 & \cdots & 0 & R(1) & R(0) & R^{t}(1) \\
0 & 0 & \cdots & 0 & R(0) & R^{t}(1)
\end{array}\right]
$$

with each individual block being defined as

$$
R_{k l}(m)=\int A_{k} s_{k}\left(t-\tau_{k}\right) A_{l} s_{l}(t+m T-\eta) d t .
$$

It has been proved (see $[11,16])$ that the outputs of a bank of $K$ matched filters provide sufficient statistic to estimate the information bits

$$
b=\left[b^{t}(1), b^{t}(2), \cdots, b^{t}(N)\right]^{t} .
$$

\section{SUBOPTMMAL DETECTORS}

The MLE detector [16] tries to find out the optimum sequence of information bits, $b$, that best fits eqn(1). If the information bits are assumed to be BPSK, this is an integer optimization problem and requires exponential complexity. Since in wireless voice communication, data has to be decoded in real time, this solution is unacceptable.

The suboptimal detectors on the other hand try to solve the system of linear equations given by eqn(1). In the decorrelating detector [10] the information bits are estimated from the transformed system

$$
\hat{b}=R_{N}^{-1} y=b+R_{N}^{-1} \eta .
$$

In the MMSE detector [17] the solution is obtained by the linear transformation $\hat{b}=A y$, where $A$ is chosen to minimize the mean square error $E\left[(b-A y)^{t}(b-A y)\right]$. It can be shown that this minimization is achieved for

$$
\hat{b}=\left(R_{N}+\frac{1}{2} N_{0} I\right)^{-1} y,
$$

where $N_{0}$ is the noise covariance matrix. The linear feedback detector proposed by [6] uses Cholesky decomposition of the correlation matrix as

$$
y=\underbrace{L^{t} L}_{R_{N}} b+\eta
$$

followed by the solution of two lower triangular systems of equations. Each of the above methods of computation in their naive form requires an $O\left((N K)^{3}\right)$ computational complexity. Although it is a significant improvement over the exponential cost, it is not necessarily optimal in computation. Significant improvement can be made by exploiting the structure of the system.

Most of the suboptimal multi-user detectors are concerned with the solution of a linear system and our aim is to reduce the complexity of he solution. Since the decorrelating detector perfectly embodies the principal ideas, we will restrict ourselves to this detector only. The results can be easily extended to MMSE type of detectors also.

\subsection{Use of iterative techniques}

As mentioned above the naive detection schemes do not exploit the structure of the system. For instance the correlation matrix is symmetric and block-Toeplitz. Recent advances in numerical linear algebra strongly suggest the use of iterative techniques to solve structured systems of equations. Of particular interest is the conjugategrodient(CG) method [7] to solve a symmetric system.

The multistage detector [14] uses the simplest form of iterative techniques to solve a system of equations.

In the iterative technique to solve $A x=b$ we:

- Form an initial guess of the solution vector $\tilde{x}$,

- Calculate the error $e=A x-0$,

- Update the estimate of the solution from the previous value of the solution and the error.

The system converges when the error becomes insignifcant. The rate of convergence of the system is governed by the spread of spectrum of the system. We will define a few terms from the literature [2] which will help us in better understanding of the problem.

Definition 3..1 (Spectrum) The spectrum of a system is defined as the distance between the smallest and the largest eigenvalue of the system.

Definition 3..2 (Clustered spectrum) The system defined by the matrix $A_{n}$ is said to have a clustered spectrum if for a given $n_{1}$ and $\epsilon, \exists n_{2}$ such that $\forall n \geq n_{2}$ the number of eigenvalues lying outside a spectrum of width $\epsilon$ is less than $n_{1}$.

The convergence of the conjugate gradient method depends on the condition number of the system as well as how clustered the spectrum is. For a system with non-clustered spectrum the convergence rate can be estimated as (see [1])

$$
\frac{\left\|e_{q}\right\|}{\left\|e_{0}\right\|} \leq 2\left(\frac{\sqrt{\lambda_{\max }\left(A_{n}\right)}-\sqrt{\lambda_{\min }\left(A_{n}\right)}}{\sqrt{\lambda_{\max }\left(A_{n}\right)}+\sqrt{\lambda_{\min }\left(A_{n}\right)}}\right)^{q},
$$

where $e_{q}$ is the error vector in the $q^{\text {th }}$ iteration, $\lambda\left(A_{n}\right)$ are the eigenvalues of the system and $\|x\| \equiv x^{*} A_{n} x$. In the case of a Toeplitz matrix, the eigenvalues are equally distributed and hence the system does not have a clustered spectrum [8]. This makes a Toeplitz system unsuitable for naive conjugate gradient techniques. Thus though the number of iterations required for convergence is constant, this constant is rather large for Toeplitz or 
Toeplitz-like systems. This suggests that use of the conjugate gradient method in its vanilla form to solve the system may not be the most effective.

\subsection{Preconditioned system}

The convergence rate can be increased if we can improve the condition number of the underlying system, by preconditioning the system. In this method, instead of solving the system $A_{n} x=6$ we solve the precondi-
tioned system

$$
P^{-1} A_{n} x=P^{-1} b,
$$

where $P$ is the preconditioner.

However to achieve the maximum benefit, the preconditioner should satisfy the following criteria:

- $P$ should be easy to construct,

- The preconditioner should be easily invertible, - The combined spectrum of $P^{-1} A_{n}$ should be clus-
tered.

To develop an estimate of the computational complex ity of a preconditioned conjugate gradient method, we $k^{t h}$ at the calculations done at each iteration. In the $k^{\text {th }}$ iteration the following computations are done (see
[7] for details):

- Solution of the system $P z_{k}=r_{k}$,

- Update of the solution vector $x_{k+1}$ from earlier values of the residue vectors $r_{k}$ and $z_{k}$ which involves the inner product of two vectors,

- Calculation of the residue vector $r_{k+1}=A_{n} x_{k+1}-b$.

The iteration stops when the residue vector becomes small. Each iteration involves:

- Inner products of two vectors - which is $O(n)$ in
complexity,

- Solution of a linear system, and

- A matrix vector product which is usnally $O\left(n^{2}\right)$ in

Hence each iteration needs at least $O\left(n^{2}\right)$ operations. In our correlation matrix the dimension of the matrix is $n=$ $N K$. If the system is well-conditioned only a constant number of iterations are required to reach the solution. However this scheme does not utilize the fact that the correlation matrix is block-Toeplitz. In the subsequent sections we will use the structure of the system to reduce the complexity of the system further.

\section{USING CIRCULANT MATRICES TO REDUCE COMPLEXITY}

If we use a diagonal matrix as a preconditioner, the step $P z_{k}=r_{k}$ requires linear lime. Thus the bottleneck in reducing the complexity in each iteration step is the matrix-vector product, which normally requires $O\left(n^{2}\right)$ steps. However if we consider a Toeplitz system, we can reduce the cost of the matrix vector product.
Definition 4..I (Circulant matrix) $A$ matrix $C_{n}$ is
said to be circulant if

$$
C_{n}=\left[\begin{array}{ccccc}
c_{0} & c_{-1} & \cdots & c_{2-n} & c_{1-n} \\
c_{1} & c_{0} & c_{-1} & \cdots & c_{2-n} \\
c_{2} & \ddots & \ddots & \cdots & \ddots \\
c_{n-1} & c_{n-2} & \cdots & c_{1} & c_{0}
\end{array}\right],
$$

where $c_{-i}=c_{n-i}$.

An interesting property of circulant matrices is that the columns of the Fourier matrix of order $n$ are the eigenvectors of circulant matrices; i.e

$$
C_{n}=F_{n}^{*} \Lambda F_{n},
$$

where $\Lambda$ is a diagonal matrix of eigenvalues of $C_{n}$ and $F_{n}$ is the Fourier matrix [5].

Lemma 1 The product of a Toeplitz matrix with a vec tor can be done in $O(n \log n)$ steps.

Proof: We can embed an $n \times n$ Toeplitz matrix in a circulant matrix of size at most $2 n$. A circulant matrix are colvely defined by one row the rest of the rows are circular shifts of that row. We can form the first row by augmenting the first row of the Toeplitz matrix with the first column of the matrix in the reverse order. As a
numerical example

$$
\left[\begin{array}{llll}
1 & 2 & 3 & 0 \\
4 & 1 & 2 & 3 \\
5 & 4 & 1 & 2 \\
0 & 5 & 4 & 1
\end{array}\right] \mapsto\left[\begin{array}{llllll}
1 & 2 & 3 & 0 & 5 & 4 \\
4 & 1 & 2 & 3 & 0 & 5 \\
5 & 4 & 1 & 2 & 3 & 0 \\
0 & 5 & 4 & 1 & 2 & 3 \\
3 & 0 & 5 & 4 & 1 & 2 \\
2 & 3 & 0 & 5 & 4 & 1
\end{array}\right]
$$

Now to calculate the matrix vector product $A_{n} x$, where $A_{n}$ is a Toeplitz matrix, we compute the embedded matrix vector product

$$
C_{n} \underbrace{\left[\begin{array}{ll}
x & n
\end{array}\right]^{t}}_{y}=\left[\begin{array}{cc}
A_{n} & B^{*} \\
B & V
\end{array}\right]\left[\begin{array}{l}
x \\
0
\end{array}\right]=\left[\begin{array}{c}
A_{n} x \\
w
\end{array}\right],
$$

where $C$ is a circulant matrix. Hence

$$
C_{n} y=F^{*} \Lambda F y
$$

can be calculated using two Fourier transforms of order at most $2 n$. However the FFT can be done in $O(n \log n)$ operations [4]. Thus the matrix vector product can be done in $\mathrm{O}($ nlogn $)$ steps.

Moreover if we use circulant matrices as preconditioners $[3,12]$, we can solve the circulant system in $O$ (nlogn) step. Thus each iteration for a Toeplitz system requires
less computation.

\subsection{Solution of block-Toeplitz system}

However the correlation matrix in eqn(1) is blockToeplitz rather than simply Toeplitz. We can extend the idea of preconditioned conjugate gradient methods to the block-Toeplitz case. 
Point circulant preconditioners [13] have been pro posed for any matrix, which computes the the best possible circulant preconditioners for any asymmetric matrix and we can use such \& preconditioner for the matrix $R_{N}$. But this does not utilize the block structure of the correlation matrix. Let us consider a general block-Toeplitz matrix

$$
A=\left[\begin{array}{cccc}
A_{0} & A_{1} & \cdots & A_{N-1} \\
A_{-1} & A_{0} & \cdots & A_{N-2} \\
\vdots & \ddots & \ddots & \vdots \\
A_{1-N} & A_{2-N} & \cdots & A_{0}
\end{array}\right],
$$

where each of the blocks $A_{i}$ is $K \times K$. We define a block circulant preconditioner for the matrix $A$ as

$$
c(A)=\left[\begin{array}{cccc}
C_{0} & C_{1} & \cdots & C_{N-1} \\
C_{-1} & C_{0} & \cdots & C_{N-2} \\
\vdots & \ddots & \ddots & \vdots \\
C_{1-N} & C_{2-N} & \cdots & C_{0}
\end{array}\right],
$$

where $C_{i}=c\left(A_{i}\right)$ is the point circulant preconditioner for the block $A_{i}$.

In the conjugate gradient system we also need to calculate the product $A x$ where $A$ is a block-Toeplitz matrix. However the block-Toeplitz matrix $A$ can be converted to a Toeplitz-block matrix using an appropriate permutation matrix $P$

$$
\tilde{A}=P^{*} A P=\left[\begin{array}{cccc}
\tilde{A}_{1,1} & \tilde{A}_{1,2} & \cdots & \tilde{A}_{1, K} \\
\tilde{A}_{2,1} & \tilde{A}_{2,2} & \cdots & \tilde{A}_{2, K} \\
\vdots & \vdots & \cdots & \vdots \\
\tilde{A}_{K, 1} & \tilde{A}_{K, 2} & \cdots & \tilde{A}_{K, K}
\end{array}\right] \text {, }
$$

where $\left[\tilde{A}_{i, j}\right]_{k}=\left[A_{k}\right]_{i, j}$ and each of the blocks $\tilde{A}_{i, j}$ is Toeplitz.

Thus the product can be written as

$$
A x=P\left(P^{*} A P\right) P^{*} x=P \tilde{A} \tilde{x},
$$

where the sub-products $\tilde{A}_{i, j} \tilde{x}_{i}$ involve product of a Toeplitz matrix with a vector and can be done using FFT's.

The original product $A x$ which required $\mathrm{O}\left((N K)^{2}\right)$ operation can now be done in $\mathrm{O}\left(K^{2} N \log N\right)$ operations using this transformation ( $K^{2}$ FFT-s). This is the most dominant computation in each iteration step and if the number of iterations is constant - which is the case for a properly preconditioned system - the complexity reduces from $\mathrm{O}\left((N K)^{3}\right)$ to $\mathrm{O}\left(K^{2} N \log N\right)$ operations.

\section{OPTIMAL DETECTORS}

Although using the pre-conditioned iterative techniques and exploiting the structure of the matrix we have significantly reduced the computational complexity of the system, we may not have reached the optimal algorithm. The following lemma determines the lower bound of an optimal algorithm for a block-Toeplitz system.

Lemma 2 The optimal algorithm to solve a $N K$ blockToeplitz system, where each block is $K \times K$ has a lower complexity bound of $O\left(N K^{2}\right)$.
Proof: In an $N K \times N K$ black Toeplitz matrix with block size $K$ there can be $N$ different blocks each of size $K$. Thus the minimum representation of such a block Toeplitz system requires $N K^{2}$ elements. Thus any algorithm which solves the system has to read at least these $N K^{2}$ elements and hence cannot have complexity lower than $N K^{2}$.

In the following subsections we propose two algorithmo which can achieve this lower bound.

\subsection{Algorithm 1}

The correlation matrix can be written as

$$
\begin{aligned}
\operatorname{ccc}_{N}= & \underbrace{\left[\begin{array}{ccccc}
R(0) & R^{t}(1) & 0 & \cdots & R(1)^{t} \\
R(1) & R(0) & R^{t}(1) & \cdots & 0 \\
\vdots & \vdots & \vdots & \vdots & \vdots \\
0 & \cdots & R(1) & R(0) & R^{t}(1) \\
R(1) & \cdots & 0 & R(0) & R^{t}(1)
\end{array}\right]} \\
& -\underbrace{\left[\begin{array}{ccccc}
0 & 0 & 0 & \cdots & R 1 \\
0 & 0 & 0 & \cdots & 0 \\
\vdots & \vdots & \vdots & \vdots & \vdots \\
0 & 0 & 0 & \cdots & 0 \\
R 1^{t} & 0 & 0 & \cdots & 0
\end{array}\right],}_{R_{N_{2}}}
\end{aligned}
$$

where $R_{N_{1}}$ is a block circulant matrix.

Definition 5..1 (Striped diagonal matrix) $A$ matrix is said to be striped diagonal if only the $(\mathrm{km})^{\text {th }}$ diagonal entries are nonzero, where $k=[\cdots,-1,0,1, \cdots]$ and $m$ is a positive integer.

Lemma 3 If $A$ is a block circulant matrix of onder $N K \times N K$, where each of the blocks is of size $K$, then it can be transformed to a block diagonal matrix with each block of onder $N$.

Proof: We can convert a block circulant matrix of order $N K$ with each block of size $K$ to a striped diagonal matrix by using Fourier transformation, with the diagonals $N$ apart. This is a direct cousequence of the fact that a block shift matrix is striped-diagonalized by the Fourier transform and a block-circulant matrix is a weighted sum of various block shift matrices [5].

$$
F^{*} A F=\left[\begin{array}{cccc}
D_{1,1} & D_{1,2} & \cdots & D_{1, K} \\
D_{2,1} & D_{2,2} & \cdots & D_{2, K} \\
\vdots & & \ddots & \vdots \\
D_{K, 1} & D_{K, 2} & \cdots & D_{K, K}
\end{array}\right]
$$

where $D_{i, j}$ is an $N \times N$ diagonal matrix.

A simple permutation can convert this striped diagonal matrix to a block diagonal matrix,

$$
\tilde{D}=P^{t} D P=\left[\begin{array}{cccc}
\tilde{D}_{1,1} & 0 & \cdots & 0 \\
0 & \tilde{D}_{2,2} & \cdots & 0 \\
\vdots & & \ddots & \vdots \\
0 & 0 & \cdots & \tilde{D}_{N, N}
\end{array}\right]
$$

where $\left[\tilde{D}_{i, i}\right]_{j, k}=\left[D_{j, k}\right]_{i, i}$ 
Corollary The eigenvalues of the matrix $A$ is the union of the eigenvalues of the blacks $\tilde{D}_{i, i}$.

Proof: Let $(\Lambda, V)$ be the eigen-pairs of $A$. Then

$$
A V=V \Lambda \text {. }
$$

We know, since $F^{\star} F=I$ and $P^{t} P=I$,

$$
D\left(F^{*} V\right)=\left(F^{*} A F\right) F^{*} V=\left(F^{*} V\right) \Lambda .
$$

Thus $D$ and $A$ have same eigenvalues. Furthermore

$$
\tilde{D}\left(P^{t} F^{*} V\right)=\left(P^{t} D P\right) P^{t}\left(F^{*} V\right)=\left(P^{t} F^{*} V\right) \Lambda .
$$

And since $\bar{D}$ is Bock diagonal its eigenvalues are the same as the union of the eigenvalues of the individual blocks.

However we want to solve a system $R_{N} b=y$. We can express the system as

which can be simplified as

$$
\left(P^{t}\left(F^{*} R_{N} F\right) P\right)\left(P^{t} F^{*} b\right)=P^{t} F^{*} y,
$$

$$
\tilde{D}\left(P^{t} F^{*} b\right)=P^{t} F^{*}\left(y+R_{N_{2}} b\right) .
$$

Since $\tilde{D}$ is block diagonal, the problem has reduced to the solntion of $N$ independent systems of order $K \times K$.

If we have $b_{1}=b_{N}=0$, then $R_{N_{2} b}=0$. Even otherwise we can solve the system using iterative techniques. The iterative methods converge faster when we have a very good initial estimate. We can use the output of the matched filter as the initial guess of the information bits $b$. We can then use the idea of Multistage detectors to solve the $N$ systems.

Each iteration requires $O\left(K^{2}\right)$ operations and simulation shows we need only a constant number of iterations. Thus solution of each of the systems requires $O\left(K^{2}\right)$ operations. The Fourier transform takes $O(N K \log (N K))$ operations and thus the solution of the entire system takes $\mathrm{O}\left(N K^{2}\right)+\mathrm{O}(N K \log (N K))$ steps. Since according to Lemma 2 the optimal algorithm is $O\left(N K^{2}\right)$ in complexity this algorithm achieves the lower bound.

\subsection{Algorithm 2}

Even though Algorithm 1 is optimal in time complexity it has a companion term $\mathrm{O}(N K \log N K)$. In the next algorithm we try to reduce the complexity of this companion term.

An upper shift matrix is defined as

$$
s=\left[\begin{array}{ccccc}
0 & 1 & 0 & \cdots & 0 \\
0 & 0 & 1 & \cdots & 0 \\
\vdots & \vdots & & \ddots & \vdots \\
0 & 0 & 0 & \cdots & 1 \\
1 & 0 & 0 & \cdots & 0
\end{array}\right]
$$

and a circulant matrix $C$ (see Definition 4.1) can be expressed as $\sum s^{i} c(i)$. The Fourier transform diagonalizes an upper shift matrix, i.e $F^{*} s F$

Extending this idea to block-circulant matrix we can rewrite a part of the correlation matrix $R_{N_{2}}$, which is a block circulant matrix as

$$
R_{N_{\mathrm{i}}}=\sum_{i=-1}^{1} s^{i} \otimes R(i)
$$

where denotes the Kronecker tensor product and $R(-i)=R(i)^{t}$

The Kronecker tensor product [9] of an $m \times n$ matrix $A$ and a $p \times q$ matrix $B$ is an $m p \times n q$ matrix given by,

$$
A \otimes B=\left[\begin{array}{cccc}
A_{1,1} B & A_{1,2} B & \cdots & A_{1, n} B \\
\vdots & & \cdots & \vdots \\
A_{m, 1} B & A_{m, 2} B & \cdots & A_{m, n} B
\end{array}\right] .
$$

We will use the following two results in our algorithm

- $\left(A_{1} \otimes B_{1}\right)\left(A_{2} \otimes B_{2}\right)=\left(A_{2} A_{2}\right) \otimes\left(B_{1} \otimes D_{2}\right)$ provided both the matrix products are defined.

- $(A \otimes B)^{-1}=A^{-1} \otimes B^{-1}$ provided the inverse exists. We can rewrite eqn(1) using eqn (3) and the above results
as

$$
R_{N_{1}} b=\sum_{i=-1}^{1}\left(s^{i} \otimes R(i)\right) b=y+R_{N_{2}} b .
$$

Premultiplying by $F^{*} \otimes I$, where $F$ is an order $N$ Fourier matrix and $I$ is a $K^{\text {th }}$ order identity matrix, we get

$$
\sum(\left(F^{*} \otimes I\right)\left(s^{i} \otimes R(i)\right) \underbrace{\left.(F \otimes I)\left(F^{*} \otimes I\right)\right)}_{I_{N K}} b=\left(F^{*} \otimes I\right)\left(y+R_{N_{2}} b\right) \text {. }
$$

Replacing $\left(F^{*} \otimes I\right) y$ by $\tilde{y}$ and $\left(F^{*} \otimes I\right) b$ by $\tilde{b}$ we get the simplified equation

$$
\sum\left(\left(F^{*} s^{i} F\right) \otimes R(i)\right) \tilde{b}=\tilde{y}+\tilde{R}_{N_{2}} \tilde{b} .
$$

Now if the diagonal matrix

$$
D=F^{*} s F
$$

then

$$
F^{*} s^{i} F=F^{*} s F F^{*} s^{i-1} F=\cdots=D^{i}
$$

and hence eqn(4) reduces to

$$
\sum\left(D^{i} \otimes R(i)\right) \tilde{b}=\tilde{y}+\tilde{R}_{N_{2}} \tilde{b} .
$$

This is a block diagonal system. Thus the solution of an $N K \times N K$ system is reduced to the solution of $N$ $(K \times K)$ systems. However, the advantage of this algorithm over the previous algorithm is that the Fourier transforms are of order $N$ and hence the total system can be solved in $O\left(N K^{2}\right)+O(K N \log N)$.

\subsection{Results}

We have performed extensive simulation and analysis of these two algorithms and compared the results with the multistage detector (with output of matched filter as the first stage guess) and the decorrelating detector. For simulation we have used 8 or 16 users and block sizes of 16,32 and 64 . The results are the average of bit-error probability over 50 simulations for the weakest user. The strongest user is $20 \mathrm{~dB}$ stronger than the desired user. We used SNR of $8 \mathrm{~dB}$.

The results show that both the algorithms need very few iterations (2-4) to achieve the desired accuracy in the result.This further justifies our claim that the iterative schemes with a good initial guess will converge to the solution in a constant number of iterations. 


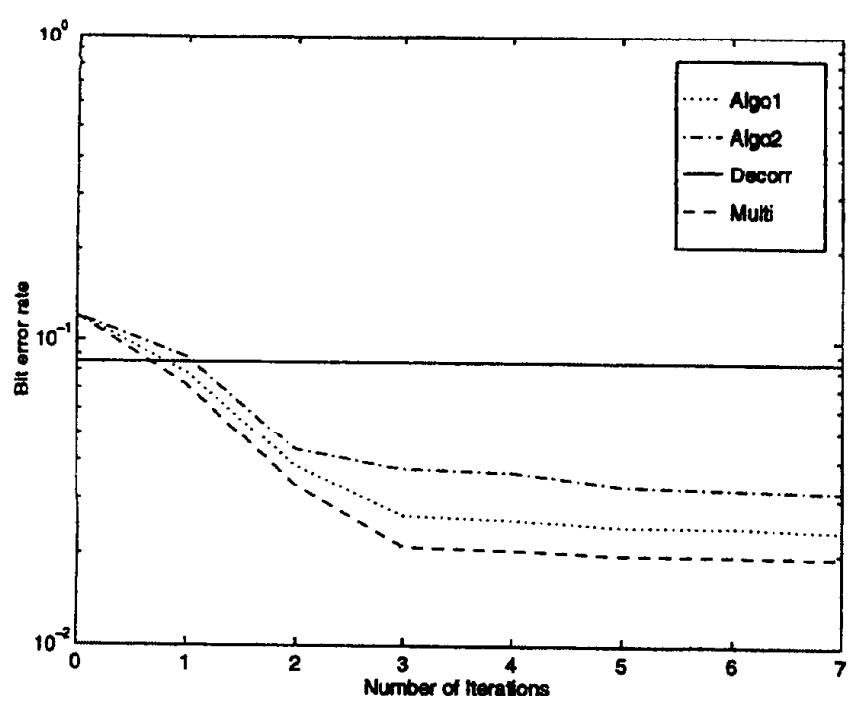

Figure 1. Comparative study of the two algorithms with Decorrelating and Multistage detectors

The results show that both the algorithms, which sentially utilize the decorrelating detector idea, perform even better than the naive decnrrelating detector. This is because for each of the individual sub blocks we are using the idea of a multistage detector. Both the algarithms tend to the results of the multiuser detector for higher iterations. Also Algorithm 1 performs better than Algorithm 2 numerically. This is because of the fact that the Fourier transform is done over larger sizes of blocks.

\section{CONCLUSION AND FUTURE WORK}

The exponential complexity of optimal multiuser detectors necessitates the use of suboptimal detectors. Most of the sub-optimal detectors in the naive implementation scheme require $\mathrm{O}\left((N K)^{3}\right)$ operations to solve a linear system involving the correlation matrix. However iterative techniques, which exploit the structure of the system, have the potential to outperform these naive schemes computationally. This paper shows that clever utilization of the structure of the correlation matrix can drastically reduce the complexity of the detectors.

In this paper we have shown how use of circulant matrices can reduce complexity of block-Toeplitz solvers. We have identified the lower bound of algorithms to solve a block Toeplitz system. We have also proposed two algorithms which achieve this lower bound.

One point that we have not emphasized in this paper is the fact that all the above algorithms can be easily parallelized. We are currently looking into the parallelization and implementation issues of these algorithms.

\section{ACKNOWLEDGMENTS}

This work was supported in part by Nokia Corporation, by the Texas Advanced Technology Program under grant \#003604-049, and by NSF under grant NCR 9506681.

\section{REFERENCES}

[1] O. Axelsson and V. Barker. Finite Element Solution of Boundary Value Problems, Theory and Computation. Academic Press, Orlando, FL, 1984.

[2] R. H. Chan and M. K. Ng. Conjugate Gradient Methods for Toeplitz Systems. SIAM Review, 38(3):427-482, September 1996.

[3] T. Chan. An Optimal Circulant Preconditioner for Toeplitz Systems. SIAM J. Sci. Stat. Comput., 9:766-771, 1988.

[4] J. Cooley and J. Tukey. An Algorithm for the Machine Calculation of Complex Fourier Series. Mathematical Computation, 19:297-301, 1965.

[5] P. Davis. Circulant Matrices. John Wiley, New York, 1979.

[6] A. Duel-Hallen. Decorrelating Decision Feedback Multiuser Detector for Synchronus Code-Division Multiple-Access Channel. IEEE Transactions on Communications, 41(2):285-290, February 1993.

[7] G. H. Golub and C.F. Van Loan. Matrix Computations. The John Hopkins University Press, 2 edition, 1989.

[8] U. Grenander and G. Szego. Toeplitz Forms and their Applications. Chelsea, New York, 2 edition, 1984.

[9] R. A. Horn and C. R. Johnson. Topics in Matrix Analysis. Cambridge University Press, 1994.

[10] R. Lupas and S. Verdu. Linear Multiuser Detectors for Synchronous Code-Division MultipleAccess Channels. IEEE Transaction of Information Theory, 41(4):123-136, 1989.

[11] J. G. Proakis. Digital Communications. McGrawHill, 1995.

[12] G. Strang. A Proposal for Toeplitz Matrix Calculations. Studies in Applied Math., 74:171-176, 1986.

[13] E. Tyrtyshnikov. Optimal and Superoptimal Circulant Preconditioners. Siam Journal of Matrix Analyois Applications, 13:459-473, 1992.

[14] M. K. Varanasi and B. Aarhang. Multistage Detection in Asynchronous Code Division Multiple Access Communications. IEEE Transactions on Communications, 38(4):509-519, April 1990.

[15] S. Verdu. Minimum Probability of Error for Asynchronous Gaussian Multiple-Access Channels. IEEE Transactions on Information Theory, 32(1):85-96, 1986.

[16] S. Verdu. Multiuser Detection. IEEE Transactions on Automatic Control, 1990.

[17] Z. Xie, R.T. Short, and C.K. Rushforth. A Family of Suboptimum Detectors for Coherent Multiuser Communications. IEEE JSAC, 8(4):683-690, 1990. 